\title{
INTEGRABLE GEODESIC FLOWS ON SURFACES
}

\author{
MISHA BIALY
}

\begin{abstract}
We propose a new condition $\aleph$ which enables to get new results on integrable geodesic flows on closed surfaces. This paper has two parts. In the first, we strengthen Kozlov's theorem on non-integrability on surfaces of higher genus. In the second, we study integrable geodesic flows on 2-torus. Our main result for 2 -torus describes the phase portraits of integrable flows. We prove that they are essentially standard outside, what we call, separatrix chains. The complement to the union of the separatrix chains is $C^{0}$-foliated by invariant sections of the bundle.
\end{abstract}

\section{IntRoduction}

Let $\Sigma$ be a closed orientable surface of genus $p \geq 1$. Given a Riemannian metric $g$ on $\Sigma$, let $g^{t}: T_{1} \Sigma \rightarrow T_{1} \Sigma$ be the corresponding geodesic flow acting on the unite circle bundle of $\Sigma$. It is an important question for dynamics and geometry if there exists a smooth function $F: T_{1} \Sigma \rightarrow \mathbb{R}$ which is invariant under the flow, that is $F\left(g^{t} x\right)=F(x)$. In this case $F$ is classically called a (first)integral of the geodesic flow. In this case, Liouville-Arnold theorem [1] implies that any connected component $L$ of the level set $\{F=c\}$, which satisfies $\left.D F\right|_{L} \neq 0$, is a 2 -torus invariant under the flow. Moreover, the dynamics of geodesic flow on this torus is linearizable. We shall use the following:

Definition 1.1. A torus $L$ lying in a level of $F$ we will call regular, if $\left.D F\right|_{L} \neq 0$, on the other hand $L$ is singular if there exists a point on $L$ where $D F$ vanishes.

In the search of smooth integrals, one usually requires, some extra condition on $F$ which prohibits from $F$ to be essentially constant. For example, one usually requires the set of regular points of $F$ to be dense. In this case the geodesic flow is called integrable.

The following theorem was proved in 1979 by V.V. Kozlov see [16] and also [17].

Theorem 1.2. Assume the genus $p>1$. Then any smooth integral $F: T_{1} \Sigma \rightarrow \mathbb{R}$ satisfying the following two conditions must be a constant.

Date: 9 May 2009.

2000 Mathematics Subject Classification. 37J35, 37J50, 53D25, $70 \mathrm{H} 07$.

Key words and phrases. Geodesic flows, first integrals, critical points, minimal geodesics. 
(K1) F has finitely many critical values.

(K2) For a dense set of points $x \in \Sigma$ the intersection of the fibre $\pi^{-1}(x)$ with any critical level $\{F=c\}$ is at most finite or coincides with the whole fibre.

As a corollary Kozlov concluded that any real-analytic integral of the geodesic flow on surfaces of higher genus must be constant. The question on topological obstructions to the integrability of geodesic flows has found considerable interest, see e.g. the works by [23], [24], [20], [21] for generalizations to higher dimensions where various stronger assumptions on non-wildness of $F$ were proposed. On the other hand, new remarkable examples of manifolds which cannot have analytically integrable geodesic flows, but do admit a $C^{\infty}$-integrable geodesic flows were recently discovered, see the works [9], 10, 11]. However, it seems that the question if such examples exist on compact surfaces of genus greater than one is still open.

We approach this question with geometric idea which enables to relax significantly the conditions of Kozlov's theorem. On the other hand, we apply this method to the case of the 2-torus. For the 2-torus there are two known classes of metrics with integrable geodesic flows. These are rotationally symmetric metrics and, the so-called, Liouville metrics. The question if there exist other examples of integrable geodesic flows is widely open. By our method we get a nontrivial information on the phase portraits for integrable geodesic flows. We show that they are essentially standard outside, what we call separatrix chains. Namely the complement to the union of these chains is $C^{0}$ foliated by invariant sections. So all dynamical complications could be located only inside the chains.

The first ingredient of the method is the theory of minimal geodesics and rays on surfaces. It was invented by M.Morse [19] and G.Hedlund [14] and further treated in [6] and also in [2] in connection with AubryMather theory. The second ingredient uses the properties of the projections of Lagrangian torii started in [7], [5], [22] (we refer to [8] for generalizations and the references).

For the approach of this paper we shall require everywhere that the metric $g$ and the function $F$ to be of class $C^{3}$, at least. This is the minimal regularity needed in order to use the properties of projections for Lagrangian torii and also for the Morse-Sard theorem for $F$ which is used below.

In order to state the first result let me formulate the main condition.

Definition 1.3. We shall say that $F: T_{1} \Sigma \rightarrow \mathbb{R}$ satisfies condition $\aleph$, if for a dense set of $x \in \Sigma$ the intersection of the fibre $\pi^{-1}(x)$ with any connected component of the critical level $\{F=c\}$ is at most countable or coincides with the whole fibre. 
Example 1. Any function which is real-analytic with respect to momenta satisfies this condition. In particular polynomials with respect to momenta are of great interest, since in all known examples of integrable geodesic flows the integral appears to be polynomial in momenta variables.

Theorem 1.4. Suppose genus $p>1$. Then any integral $F$ of the geodesic flow satisfying condition $\aleph$ must be a constant.

Corollary 1.5. There are no real-analytic with respect to momenta integrals for geodesic flows on surface of higher genus other than constants.

Let me mention that the first condition $(K 1)$ of Kozlov's theorem is not required in theorem 1.4 .

Our next results apply for the case of Riemannian 2-torus $\mathbb{T}^{2}=$ $\mathbb{R}^{2} / \mathbb{Z}^{2}$

Theorem 1.6. Let $\Sigma=\mathbb{T}^{2}$. Let $F$ be a non-constant integral of the geodesic flow satisfying condition $\aleph$. Then there exists an invariant 2torus $L$ of the geodesic flow lying in the regular level of $F$ which is a smooth section of $T_{1} \mathbb{T}^{2}$.

In fact we can specify in the following way:

Corollary 1.7. The torus $L$ in the theorem can be chosen in such a way that all the orbits of the flow on $L$ project to minimal closed geodesics of the same homotopy type.

One can deepen the theorem 1.6 in the following way, which can be interpreted as non-existence of the "instability" zones:

Theorem 1.8. Let $F$ be a non-constant integral of the geodesic flow satisfying condition $\aleph$. Let $N$ be a domain in $T_{1} \mathbb{T}^{2}$ bounded by two disjoint sections $L_{1}, L_{2}$ which are singular invariant torii of the geodesic flow. Then there exists a regular torus $L$ lying inside $N$ which is a section invariant under the flow.

Notice that the claim of this theorem obviously holds true if one of the boundary torii $L_{1}, L_{2}$ is regular. Because one can push it inside $N$ by a flow $v^{t}$ of the vector field $v=\nabla F /|\nabla F|^{2}$ (see proof of theorem 1.10 below). Moreover, assuming theorem 1.6 one can start the following process. Take a regular torus $L$ and move it with the flow $v^{t}$ inside $T_{1} \mathbb{T}^{2}$ as long as possible in a positive and negative direction. This cannot be continued when the torii become singular. Then, by theorem 1.8 between any two singular torii one can take a new regular torus and to flow it again, and so on. The process terminates when one gets singular torii touching one another. To be more precise, we introduce the following 
Definition 1.9. By a separatrix chain we mean a closed subset $X \subset$ $T_{1} \mathbb{T}^{2}$ bounded by two different singular torii $L_{+}, L_{-}$such that both of them are Lipshits sections invariant under the geodesic flow such that the intersection $L_{+} \cap L_{-}$equals the union of all periodic minimizing trajectories of a common rational rotation number. No other invariant sections are allowed to pass inside $X$.

Theorem 1.10. Let $F$ be a non-constant integral of the geodesic flow of the 2-torus satisfying condition $\aleph$. There are at most countably many separatrix chains. Through any point in the complement of their union passes a unique invariant section which is either regular or singular. In case it is singular, it is a limit from both sides of regular invariant sections. The complement to the union of separatrix chains is $C^{0}$ foliated by invariant sections.

For example, there are no separatrix chains at all if the metric is flat (and in fact only in this case, by a theorem of E.Hopf [15]). There are precisely two chains for rotationally symmetric Riemannian metrics and four of them for Liouville metrics. The number of these separatrix chains (it is always even, due to the symmetry of the metric) corresponds to a number of non-smooth points for the ball of stable norm on $H_{1}\left(\mathbb{T}^{2} ; \mathbb{R}\right)$. This connection with the stable norm follows from Bangert's paper [3] (see also paper by Mather [18]).

Corollary 1.11. If the integral $F$ is assumed to be real-analytic in momenta then there are at most finitely many of separatrix chains.

This is because the function $F$ has equal values on $L_{+}$and $L_{-}$and therefore the derivative along the fibre of $F$ must vanish somewhere in between. By analyticity this can happen only finitely many times.

The organization of the paper is as follows. In Section 2 we summarize the needed facts on minimal geodesics and Lagrangian projections of invariant torii. In Section 3 we prove that no minimal rays can be trapped "inside" compressible invariant tori. Section 4 contains the proofs of main theorems 1.4, 1.6. In Section 5 theorems 1.8 and 1.10 are proved. The last Section 6 contains facts on critical points of $F$ (not necessarily satisfying condition $\aleph$ ).

\section{ACKNOWLEDGEMENTS}

I am thankful to Leonid Polterovich, with whom we started the study of Lagrangian torii and Minimal geodesics many years ago. During these years we discussed the subject many times and I learnt a lot of things from him.

\section{Minimal GeOdesics AND TORII}

The proof of our main results relies on interplay between theory of minimal geodesics on surfaces and theory of Lagrangian singularities 
of projections of invariant torii. Let me summarize the needed facts in several theorems below.

Let us represent the surface as a quotient $\Sigma=\widetilde{\Sigma} / \Gamma$ where $\Gamma$ is the fundamental group of the surface acting on the covering by isometries. Here $\widetilde{\Sigma}$ is a Euclidian plane $\mathbb{R}^{2}$ for $p=1$, and the Poincare unite disc $\mathbb{D}$ for $p>1$. We shall lift the metric $g$ to the covering and also denote by $g_{0}$ the Euclidian and Hyperbolic metric on $\widetilde{\Sigma}$ respectively. We use the following terminology. An isometric image of $\gamma:(-\infty,+\infty) \rightarrow \widetilde{\Sigma}$ will be called a minimal geodesic while an isometric image of $\gamma:[0,+\infty) \rightarrow$ $\widetilde{\Sigma}$ will be called a ray starting at $\gamma(0)$. Projections of minimal geodesics and rays from $\widetilde{\Sigma}$ to $\Sigma$ form by definition a class of minimal geodesics and rays on $\Sigma$. The orbits of geodesic flow corresponding to minimal geodesics and rays we will call by minimal orbits. It was proved by Morse that each minimal geodesic stays in a finite distance from a unique $g_{0}$-geodesic on the covering $\widetilde{\Sigma}$ which is called the type of the minimal geodesic. The type is determined by the slope of the straight line on the Euclidian plane for $p=1$, and by the end points of the Hyperbolic geodesic for $p>1$. A minimal geodesic is called to be of periodic type if the corresponding $g_{0}$-geodesic becomes closed being projected to $\Sigma$, and it has a non-periodic type, in the opposite case. We shall use the following:

\section{Theorem 2.1.}

1. For any point $x \in \widetilde{\Sigma}$ there are uncountably many rays starting at $x$. They are parameterized by the slope for the case $p=1$, and by points of the ideal circle in the case $p>1$. This means that for the case $p=1$, every such a ray has a given slope. And in the case $p>1$ such a ray stays on a bounded distance at infinity from any Hyperbolic geodesic approaching a given point of the ideal circle.

2. Some of these rays are of periodic type. More precisely, for any point $x \in \widetilde{\Sigma}$ there exists a ray starting at $x$ asymptotic to a minimal geodesic of a given periodic type (or the ray itself is a half of periodic minimal geodesic).

The second statement of the theorem is proved explicitly in the paper by M.Morse. The first is immediate for the case of the torus (see also [6]) and for higher genus it can be easily deduced from the theory of minimal geodesics in the following way. Given a point $x \in \widetilde{\Sigma}$ and a point $y$ on the ideal boundary, choose hyperbolic geodesics $l_{1}, l_{2}$ with one their end at $y$, such that $x$ is contained in the strip between them and lies sufficiently far away from them. Then one can construct by a limiting argument, two minimal $g$-geodesics $\gamma_{1}, \gamma_{2}$ of the type of $l_{1}, l_{2}$ respectively such that they do not intersect and contain $x$ in the strip between them. Having such a strip, one constructs a ray lying inside in a standard way. Notice that unlike the torus case where the ray can be chosen to be asymptotic to a minimal geodesic of given slope, the 
ray constructed above for higher genus stays on a bounded distance at infinity from $\gamma_{1}, \gamma_{2}$. The following fact, important for our purposes, was proved by Morse [19], for $p>1$, and by Hedlund in [14, for $p=1$. Given any two minimal periodic geodesics of the same periodic type on $\widetilde{\Sigma}$ such that there are no other minimal periodic geodesics in the strip between them, there always exist two heteroclinic connecting geodesics in the strip between them (and therefore also all their translates). It follows from this fact that the set of minimal geodesics of a given periodic type on $\widetilde{\Sigma}$ has separation zero, i.e. for any $\epsilon>0$ there exists an $\epsilon$-chain connecting them. We can summarize:

Theorem 2.2. Fix a periodic type of minimal geodesics on $\widetilde{\Sigma}$ and denote by $\widetilde{M} \subset T_{1} \widetilde{\Sigma}$ be a set of all unite tangent vectors to minimal geodesics of the fixed periodic type, let $M$ be the projection of $\widetilde{M}$ to $T_{1} \Sigma$. Then

1. The set $\widetilde{M}$ has separation zero, $M$ is a closed connected set.

2. For any point $x \in \widetilde{\Sigma}$ there exists a unite tangent vector $v$ at $x$ such that the trajectory of the geodesic flow $g^{t}(x, v)$ is approaching $\widetilde{M}$ as $t \rightarrow+\infty$.

Let me summarize now the needed facts on Lagrangian torii invariant under the geodesic flows. These results started from [6], 7] generalizing Birkhoff's first and second theorems from area preserving twist maps to the case of geodesic flows. Later they were generalized further in many other directions. We refer the reader to paper [8] for various generalizations and references. I will need also the results from 5] where compressible invariant torii were studied. I will remind first the following:

Theorem 2.3. Let $L \subset T_{1} \Sigma$ be an invariant torus of the geodesic flow. Then the set of singular points of the projection of $\left.\pi\right|_{L}$ is a union of finite number simple closed non-intersecting curves on L. They are not null-homotopic on $L$ and the trajectories of the flow intersect them transversally.

Recall that an imbedded torus $L$ inside $T_{1} \Sigma$ is called compressible if the homomorphism induced by inclusion, $i_{*}: \pi_{1}(L) \rightarrow \pi_{1}\left(T_{1} \Sigma\right)$ has a nontrivial kernel, and incompressible otherwise. Remarkably, if $L$ is an invariant torus of the geodesic flow it happens that it is either compressible or projects diffeomorhically. Moreover, one can construct the compressing disc explicitly as it is proved in [5].

Let me summarize in the following way the known results on Lagrangian torii from [7], [5].

Theorem 2.4. Let $L \subset T_{1} \Sigma$ be a an invariant torus of the geodesic flow.

1. For $p>1$ all invariant torii $L \subset T_{1} \Sigma$ are compressible. Incompressible torii may exist only for $\Sigma=\mathbb{T}^{2}$. In this case, if the dynamics 
on $L$ is chain-recurrent (this always holds for regular torii) then $L$ is a smooth section of the $T_{1} \mathbb{T}^{2}$.

2. Moreover, write $\mathbb{T}^{2}=\mathbb{R}^{2} / \mathbb{Z}^{2}$ and fix a Euclidian structure on $\mathbb{R}^{2}$ in order to trivialize the bundle $T_{1} \mathbb{T}^{2}=\mathbb{T}^{2} \times \mathbb{S}^{1}$. Then the the projection of $L$ on the $\mathbb{S}^{1}$-factor is null homotopic. So, $L$ is a graph of a smooth function $f: \mathbb{T}^{2} \rightarrow \mathbb{S}^{1}$.

3. There exists a constant $K>0$ depending only on the metric $g$ such that any continuous graph invariant under the flow is in fact $K$ Lipshits. In particular the functions of the previous item have a-priori bounded gradients: $|\nabla f| \leq K$. In addition, trajectories on the graph projects to minimal geodesics.

The first statement of this theorem is a combination of the so-called generalized second Birkhoff theorem for the case of geodesic flows together with a general idea on sections for invariant torii of geodesic flows from [5]. The third statement is the so-called generalized first Birkhoff theorem, which reflects the so called twist condition, together with the property of field of extremals. The second statement follows from a little topological argument using minimal geodesics of the periodic type. Let me remark here that it was proved in [4] that in the generalized Birkhoff theorem it is enough to assume for $L$ to be only continuous, provided there are no periodic orbits on $L$.

\section{Non-Trapping of Minimal GeOdesics.}

Now I am in position to define the "interior" components of compressible torii as follows. Let $L \subset T_{1} \Sigma$ be a smooth invariant compressible torus of the geodesic flow. Then either $L$ bounds a solid torus or $L$ is contained in a a part of $T_{1} \Sigma$ homeomorphic to a 3-ball (it is proved with the help of compressing disc, see [13]). Define accordingly the "interior" $I(L)$ to be the interior of the solid torus bounded by $L$, or the interior of the component lying inside the 3-ball. Notice that this is a correct definition. Indeed, if it happened that $L$ bounds a solid torus and some component of the complement lies in a ball, then it should be a solid torus lying in the 3-ball. Because otherwise $T_{1} \Sigma$ would be simply connected, which is not the case. Here is one of our crucial observations:

Theorem 3.1. Let $L$ be a smooth compressible invariant torus in $T_{1} \Sigma$ such that the dynamics on it is chain-recurrent (for example this always holds for regular invariant torii). Then all geodesic trajectories corresponding to minimal geodesics and rays do not lie neither on $L$ nor in $I(L)$.

Proof. Let us prove first that no minimal trajectory or ray can lie on $L$. We have $\left.\pi\right|_{L}$ is not a diffeomorphism and due to the condition of chain-recurrence we have two possible cases for the dynamics on $L$. In the first case the orbits are not closed and therefore each orbit 
must intersect the singularity curves of theorem 2.3 infinitely many times. Each such intersection increases by one the Morse index of the corresponding geodesic. Thus it can not be minimal. In the second case, all the trajectories on $L$ are closed, then all of them project to geodesics of the same length, since the torus $L$ is Lagrangian (as a torus in the (co-) tangent bundle). Some of them necessarily intersect singularity curves and therefore not minimal as explained above. But all of them have the same length, therefore all the orbits on $L$ are not minimal.

It is simple to rule out the case when $I(L)$ lies in a ball, since it cannot happen for minimal trajectory to lie in a ball. Because otherwise any lift of the minimal geodesic to the universal cover would be bounded on $\widetilde{\Sigma}$ which is impossible.

Suppose now that $I(L)$ is a solid torus. Assume by contradiction that there exists a minimal half-trajectory inside $I(L)$ which corresponds to a ray. Then there exists a point in $\omega$-limit set of this half-trajectory. The orbit of this point is a minimal orbit (by a limiting argument). This minimal orbit must lie also inside $I(L)$ because as was proved above, it can not lie on the boundary. In addition, this orbit must be of a periodic type, determined by the core of the solid torus. Then by the first statement of theorem 2.2 all minimal orbits of this periodic type must stay inside $I(L)$ and by the second statement, fiber of any point of the covering $\widetilde{\Sigma}$ can be connected by a minimal trajectory to one of them lying inside. But this is impossible since $L$ is invariant and can not be crossed.

\section{Proof of MAIN THEOREMS}

In this section we prove the theorems 1.4 and then 1.6 .

Proof of theorem 1.4. The idea is very simple. If $\Sigma$ is of genus $p>1$ then it follows from theorem 2.4 that all regular torii are compressible. Therefore, by theorem 3.1 all minimal geodesics and rays lie outside their "interior" components and belong to singular levels of the integral $F$. Denote by $R$ the class of all regular torii and define the set

$$
Z=T_{1} \Sigma-\bigcup_{L \in R} I(L)
$$

This is a compact invariant set.

Lemma 4.1. The set $Z$ is an invariant continuum, i.e. it is compact, connected invariant set.

We complete first the proof of the theorem. Notice that by the very construction, there are no regular torii left in $Z$ since deleting $I(L)$ for all regular $L$ we delete, of course, $L$ itself since nearby torii are regular either. So in particular all regular levels of $F$ are deleted. Thus, $Z$ lies in a union of all singular levels. So $F$ attains only critical values on 
$Z$. By Morse-Sard theorem (proved in this case by A.Morse [12]) for $F$ and by the lemma it follows that $F$ on $Z$ attains a unique critical value. Moreover, the set of all tangent vectors to all minimal geodesics and rays lie in $Z$ by theorem 3.1. By theorem 2.1 there are uncountably many of them in a fibre of any point $x \in \Sigma$. By condition $\aleph$ this forces $F$ to be a constant. This completes the proof of theorem 1.4.

Proof of lemma 4.1. Since the unite cotangent bundle $T_{1} \Sigma$ is secondcountable topological space then, the union $\bigcup_{L \in R} I(L)$ can be replaced by at most countable union. Therefore there exist a sequence of torii $L_{n} \in R, n \geq 1$ such that

$$
\bigcup_{L \in R} I(L)=\bigcup_{j=1}^{\infty} I\left(L_{j}\right) .
$$

Then one can write

$$
Z=T_{1} \Sigma-\bigcup_{j=1}^{\infty} I\left(L_{j}\right)=\bigcap_{n=1}^{\infty} Z_{n}
$$

where

$$
Z_{n}=T_{1} \Sigma-\bigcup_{j=1}^{n} I\left(L_{j}\right) .
$$

It is clear that each $Z_{n}$ is compact and connected and $Z_{n+1} \subseteq Z_{n}$. Thus, $Z$ is compact and connected either as an intersection of nested sequence of compact connected sets. This proves the lemma.

Proof of theorem 1.6 and corollary 1.7. We claim first that there exists a torus $L$ in $T_{1} \mathbb{T}^{2}$ which lies in a regular level of $F$ and is not compressible. Indeed, otherwise all torii of regular levels $F$ would be compressible. Then one can can define the set $Z$ and proceed exactly as in the proof of theorem 1.4, getting a contradiction with the assumption that $F$ is not a constant function. So incompressible $L$ exists. Apply theorem 2.4 in order to get that $L$ can be written as a graph of a smooth function $f: \mathbb{T}^{2} \rightarrow \mathbb{S}^{1}$ and all the orbits on $L$ are minimal geodesics. There are two cases which may occur for the dynamics on $L$ (recall $L$ is satisfies Liouville-Arnold theorem). In the first case all the orbits are closed and then we are done. In the second case geodesics of $L$ have irrational rotation number. Since $L$ lies in a regular level one can take torus $\tilde{L}$ lying in a nearby levels which are also invariant graphs consisting of minimal orbits. We claim that in the process of such perturbation the rotation number must vary. Indeed if the rotation number of $L$ and $\tilde{L}$ were the same irrational number, it would imply that two different minimal geodesics, coming from $L$ and $\tilde{L}$, with the same irrational rotation number intersect. But this is impossible. Moreover, since the rotation number is a continuous function, then this claim implies that it can be made rational by such a perturbation. 


\section{Separatrix CHAins}

In this section we prove theorems 1.8 and 1.10. We shall use heavily the ordering properties of minimal geodesics on $\mathbb{T}^{2}$. The following lemma enables to repeat the proof of theorem 1.4 for the case of theorem 1.8 .

Lemma 5.1. Let $N$ be a domain $T_{1} \mathbb{T}^{2}$ bounded by two disjoint graphs $L_{1}, L_{2}$ invariant under the geodesic flow. Then for any point $x \in \mathbb{T}^{2}$ there exist uncountably many rays, such that the corresponding halforbits lie in $N$.

Proof of the lemma. It follows from the ordering properties of minimal geodesic on the covering plane. Any two minimal geodesic of different slopes intersect exactly at one point. Moreover, two different minimal geodesics of the same slope can intersect only if their slope is rational and the geodesics are the pair of heteroclinic connections. Using this fact and the fact that the orbits lying on $L_{1}, L_{2}$ (by theorem 2.4) are minimal, one can conclude that the rotation numbers of $L_{1}, L_{2}$ must be different. Indeed, otherwise $L_{1}, L_{2}$ would have the same rational rotation number and both would contain minimal periodic geodesics. This would contradict their disjointness. Therefore, all minimal geodesics and rays starting at $x$ with slopes in the interval between the rotation numbers of $L_{1}$ and $L_{2}$ lie also in between the torii $L_{1}, L_{2}$. There are uncountably many of them. This completes the proof of the lemma.

Proof of theorem 1.10. Let $F$ be a non-constant integral of the geodesic flow. Introduce any Riemannian metric on $T_{1} \mathbb{T}^{2}$ and the vector field $v=\nabla F /|\nabla F|^{2}$, defined on an open set of regular points of $F$. Denote by $v^{t}$ the flow of $v$. For every regular point point $x$ there exists a maximal finite open interval of existence of trajectory $v^{t}(x)$. For any regular invariant torus $L$ let $(\alpha(L), \beta(L))$ be the maximal open interval of existence of $v^{t}(x)$ for all $x \in L$. At the moments $\alpha(L), \beta(L)$ some points of $L$ tend to the set of critical points. Obviously the torii $L_{t}:=$ $v^{t}(L)$ are also regular invariant torii for any,$t \in(\alpha(L), \beta(L))$. Denote by $B(L)$ the domain swept by $L_{t}$,

$$
B(L)=\bigcup_{t \in(\alpha(L), \beta(L))} L_{t} .
$$

Assume now that $L$ is a regular section. Then it follows from theorem 2.4 that all $L_{t}$ are uniformly $K$-Lipshits. Therefore it follows from Arzela-Ascoli theorem that there exist uniform limits which are also $K$-Lipshitz torii:

$$
L_{\alpha}=\lim _{t \rightarrow \alpha(L)} L_{t}, \quad L_{\beta}=\lim _{t \rightarrow \beta(L)} L_{t} .
$$

Both of the limits are singular invariant torii. Denote by $B$ the open set of all regular invariant sections, i.e $B=\bigcup_{L} B(L)$. Assume from now on 
that the integral $F$ satisfies condition $\aleph$, then by theorem 1.6 the set $B$ is not empty. Fix a regular section $L_{0}$ in $B$ for the rest of the proof. Fix an orientation on the fibres. It determines the order on $B-L_{0}$. For any regular section $L$ disjoint from $L_{0}$, there are two regions between them, $\left[L_{0}, L\right]$ and $\left[L, L_{0}\right]$, in accordance with orientation of the fibres. Let us describe the complement to $B$. For any point $P \in T_{1} \mathbb{T}^{2}-B$. Define

$$
\begin{gathered}
L_{+}=\inf \left\{L: L \in B, L \neq L_{0}, P \in\left[L_{0}, L\right]\right\}, \\
L_{-}=\sup \left\{L: L \in B, L \neq L_{0}, P \in\left[L, L_{0}\right]\right\} .
\end{gathered}
$$

By Arzela-Ascoli theorem these are $K$-Lipshits invariant sections which are singular and

$$
L_{+} \subset\left[L_{-}, L_{0}\right], \quad L_{-} \subset\left[L_{0}, L_{+}\right] .
$$

It follows by theorem 1.8 that $L_{+}, L_{-}$cannot be disjoint. It may happen that they coincide, in this case we are done. In the other case, they have a nontrivial intersection. Then the rotation number for both of them is the same, since they have common orbits, and moreover, it must be rational, because any two minimal geodesics of the same irrational rotation number can not cross each other. Therefore the intersection $L_{+} \cap L_{-}$consists of periodic minimal geodesics. It is easy to see that in fact $L_{+} \cap L_{-}$coincides with the set of all minimal periodic orbits of this rational type. This completes the proof.

\section{REMARKS ON CRITICAL POINTS OF $F$.}

This section contain some simple facts about critical points of $F$, for any smooth $F$ (not necessarily satisfying condition $\aleph$ ). Let $\gamma$ be a minimal periodic geodesic on $\Sigma$. We shall say it is deformable if it has a neighborhood filled by minimal geodesics of the same type. The following fact is simple: The trajectory of the geodesic flow corresponding to a non-deformable minimal geodesic consist of critical points of $F$. This is because, otherwise one could move the closed orbit in $T_{1} \Sigma$ by the $f^{t}$ of $F$ and get a family of closed orbits nearby. All of them project to closed minimal geodesics. If for a given periodic type not all minimal geodesics are closed then there always exist non-deformable minimal geodesic of this type. For example if $p>1$ for every periodic type there exists a non-deformable minimal geodesic. For $p=1$, given a periodic type there exist a non-deformable geodesic of a given type or there is an invariant torus, consisting of minimal periodic orbits. Having enough non-deformable minimal geodesics one can take their limits to get geodesics of other non-periodic types such that their orbits in $T_{1} \Sigma$ also consist of critical points of $F$. In such a way one proves that for $p>1$ and for any not periodic type there always exist a minimal geodesic of this type such that the corresponding orbit consists of critical points (These are so called boundary geodesics in terminology 
of Morse [19]). Probably the same is true for all recurrent orbits for non-trivial Aubry-Mather sets in the case of $\Sigma=\mathbb{T}^{2}$. We don't know however how this information can be used further.

\section{REFERENCES}

[1] Arnold, V. I. Mathematical methods of classical mechanics. Translated from the Russian by K. Vogtmann and A. Weinstein. Graduate Texts in Mathematics, 60. Springer-Verlag, New York-Heidelberg, 1978.

[2] Bangert, V., Mather sets for twist maps and geodesics on tori. Dynamics reported, Vol. 1, 1-56, Dynam. Report. Ser. Dynam. Systems Appl., 1, Wiley, Chichester, 1988.

[3] Bangert, V., Geodesic rays, Busemann functions and monotone twist maps. Calc. Var. Partial Differential Equations 2 , no. 1,(1994) 49-63.

[4] Bialy, M., Aubry-Mather sets and Birkhoff's theorem for geodesic flows on the two-dimensional torus. Comm. Math. Phys. 126 , no. 1(1989) 13-24.

[5] Bialy, M., On the number of caustics for invariant tori of Hamiltonian systems with two degrees of freedom. Ergodic Theory Dynam. Systems 11, no. 2,(1991) 273-278.

[6] Bialy, M., Polterovich, L., Geodesic flow on the two-dimensional torus and phase transitions "commensurability-noncommensurability". Funct. Anal. Appl. 20 (1986) 260-266.

[7] Bialy, M., Polterovich, L., Lagrangian singularities of invariant tori of Hamiltonian systems with two degrees of freedom. Invent. Math. 97 , no. 2 (1989) 291-303.

[8] Bialy, M., Polterovich, L., Hamiltonian diffeomorphisms and Lagrangian distributions. Geom. Funct. Anal. 2, no. 2(1992) 173-210.

[9] Bolsinov A. V., Taimanov I. A. Integrable geodesic flows with positive topological entropy. Invent Math 140 (2000) 639-650.

[10] Butler L. A new class of homogeneous manifolds with Liouville-integrable geodesic flows. C R Math Rep Acad Sci Can, 21 (1999) 127-131

[11] Butler L. New examples of integrable geodesic flows. Asian J Math 4 (2000) 515-526.

[12] Morse, Anthony, The behavior of a function on its critical set. Ann. of Math. (2) 40, no. 1,(1939) 62-70.

[13] Hatcher,A., Notes on basic 3-manifolds topology. http://www.math.cornell.edu/ hatcher

[14] Hedlund, G., Geodesics on a two-dimensional Riemannian manifold with periodic coefficients. Ann. Math. 33(1932) 719-739.

[15] Hopf, E., Closed surfaces without conjugate points. Proc. Natl. Acad. Sci. USA 34, 4751 (1948).

[16] Kozlov,V.V., Topological obstructions to the integrability of natural mechanical systems. Soviet Math. Dokl. 20 (1979) 1413-1415.

[17] Kozlov, V.V.,Integrability and non-integrability in Hamiltonian mechanics. Uspekhi Mat. Nauk 38:1 (1983) 3-67.

[18] Mather, J., Differentiability of the minimal average action as a function of the rotation number. Bol. Soc. Bras. Mat. 21 (1990) 59-70.

[19] Morse, M. A. fundamental class of geodesics on any closed surface of genus greater than one. Trans. Am. Math. Soc. 26 (1924) 25-60.

[20] Paternain G. P. On the topology of manifolds with completely integrable geodesic flows. Erg Th Dynam Sys 12(1992) 109-121. 
[21] Paternain G. P. On the topology of manifolds with completely integrable geodesic flows. II. J Geom Phys 13 (1994)289-298.

[22] Polterovich, L., The second Birkhoff theorem for optical Hamiltonian systems. Proc. Amer. Math. Soc. 113, no. 2, (1991)513-516.

[23] Taimanov I. A. Topological obstructions to integrability of geodesic flows on nonsimply connected manifolds. Izv Akad Nauk SSSR, Ser Mat, 1987, 51: 429435; translation in Math USSR Izv 30(1988)403-409.

[24] Taimanov I. A. Topology of Riemannian manifolds with integrable geodesic flows. translation in Proc Steklov Inst Math 205(1995)139-150.

Raymond and Beverly Sackler School of Mathematical Sciences, Tel Aviv University, IsRael

E-mail address: bialy@post.tau.ac.il 